\title{
Linear Multistep Methods with Reduced Truncation Error for Periodic Initial-value Problems
}

\author{
P. J. VAN DER HouWen AND B. P. SOMMEIJER \\ Centrum voor Wiskunde en Informatica, Kruislaan 413, \\ 1098 SJ Amsterdam, The Netherlands
}

[Received 5 September 1983]

\begin{abstract}
A common feature of most methods for numerically solving ordinary differential equations is that they consider the problem as a standard one without exploiting specific properties the solution may have.

Here we consider initial-value problems the solution of which is a priori known to possess an oscillatory behaviour. The methods are of linear multistep type and special attention is paid to decreasing the value of those terms in the local truncation error which correspond to the oscillatory solution components. Numerical results obtained by these methods are reported and compared with those obtained by the corresponding conventional linear multistep methods and by the methods developed by Gautschi.
\end{abstract}

\section{Introduction}

WE WILL BE CONCERNED with linear $k$-step methods

$$
\rho(E) y_{n}-h \sigma(E) \dot{y}_{n}=0
$$

for integrating the equation

$$
\dot{y}(t)=f(t, y(t))
$$

in cases where the exact, local solution is known to be approximately of the form

$$
y(t) \approx c_{0}+\sum_{j=1}^{m} c_{j} e^{i \omega_{j} t},
$$

where the frequencies $\omega_{j}$ are in the interval $[\underline{\omega}, \bar{\omega}]$ with $\underline{\omega}$ and $\bar{\omega}$ given numbers. Assuming (1.3), the values of $\underline{\omega}$ and $\bar{\omega}$ can often be derived from the eigenvalue spectrum of the Jacobian matrix $\partial f / \partial y$ (see e.g. Section 4).

In the special case where the frequencies $\omega_{j}$ in (1.3) are such that the solution is periodic or "almost" periodic, that is $y(t) \approx y\left(t+2 \pi / \omega_{0}\right)$ for some a priori given frequency $\omega_{0}$, Gautschi (1961) has developed special linear multistep methods. However, these methods are rather sensitive to a correct prediction of the frequency $\omega_{0}$ (cf. Sections 4.2 and 4.3). This unfavourable property of the Gautschi methods (which are of Adams type, i.e. $\rho(\zeta)=\zeta^{k}-\zeta^{k-1}$ ) motivated Neta \& Ford (1984) to propose methods of Nyström and Milne-Simpson type $\left(\rho(\zeta)=\zeta^{k}-\zeta^{k-2}\right)$. These methods, however, although demonstrating a less sensitive behaviour if the value of $\omega_{0}$ is perturbed, are rather sensitive to non-imaginary noise (cf. Section 4.3). 
In this paper we will try to construct methods which do not suffer the abovementioned disadvantages.

\section{Reduction of the Truncation Error for a Given Interval of Frequencies}

Let

$$
\phi(z):=\rho\left(e^{z}\right)-z \sigma\left(e^{z}\right),
$$

then the local truncation error at $t_{n+k}$ is given by (cf. e.g. Lambert, 1973, p. 27)

$$
\mathrm{T}_{n+k}:=\left.\phi\left(h \frac{d}{d t}\right) y(t)\right|_{t=t_{n}},
$$

where $y(t)$ denotes the exact solution satisfying $y\left(t_{n}\right)=y_{n}$. We assume $\phi(0)=0$; in the case (1.3) we then have approximately

$$
\left|\mathbf{T}_{n+k}\right| \leqslant \sum_{j=1}^{m}\left|c_{j}\right|\left|\phi\left(i v_{j}\right)\right|, \quad v_{j}:=\omega_{j} h
$$

In the case where $y(t)$ is a periodic or "almost" periodic function with frequency $\omega_{0}$, we may replace $y(t)$ by the approximating Fourier series

$$
y(t)=\sum_{l=0}^{\infty} \tilde{c}_{l} e^{i l \omega_{0} t}+\varepsilon(t), \quad|\varepsilon| \ll 1
$$

to obtain the approximate inequality

$$
\left|\mathbf{T}_{n+k}\right| \leqslant \sum_{l=0}^{\infty}\left|\tilde{c}_{l}\right|\left|\phi\left(i l v_{0}\right)\right|, \quad v_{0}:=\omega_{0} h .
$$

The inequalities (2.3) and (2.5) suggest essentially three approaches for adapting the linear multistep method to the additional information available on the exact, local solution $y(t)$. Let us start with a family of linear $k$-step methods containing $2 q$ not yet specified coefficients, and let the remaining coefficients be such that $\phi(z)=O\left(z^{r}\right), r \geqslant 1$. Then one may proceed as indicated in Table 1 .

The first approach is that of Gautschi. The resulting method is said to be of trigonometric order $q$. Its order in the conventional sense (the algebraic order) is given by $p=2 q$. The Gautschi method may be interpreted as a method which is exponentially fitted at the points $i l \omega_{0}, l=1, \ldots, q$ (cf. Liniger \& Willoughby (1970).

Many authors proposed integration methods following the second approach. For

TABLE 1

Reduction of truncation errors

\begin{tabular}{cll}
\hline I $y(t)$ is periodic with frequency $\omega_{0}:$ & Solve the system (Gautschi, 1961) \\
& $\phi\left(i l \omega_{0} h\right)=0, l=1, \ldots, q$ \\
II $y(t)$ has dominant solution components & Solve the system \\
$\exp \left(i \omega_{j} t\right)$ with given $\omega_{j}:$ & $\phi\left(i \omega_{j} h\right)=0, j=1, \ldots, q$ \\
III $y(t)$ has dominant solution components & Try to minimize the function (cf. (2.7)) \\
$\exp (i \omega t)$ with $\omega \in[\underline{\omega}, \bar{\omega}]:$ & $|\phi(i \omega h)|$ on $\underline{\omega} \leqslant \omega \leqslant \bar{\omega}$
\end{tabular}


example. Stiefel \& Bettis (1969), Bettis (1970) and Lyche (1972) constructed schemes by which not only the harmonic oscillations $\omega_{j}$ are integrated exactly but by which also products of Fourier and ordinary polynomials are integrated without truncation error. However, to apply this approach we should start with a linear multistep method containing sufficiently many free parameters in order to achieve that $\phi\left(i \omega_{j} h\right)=0$ for all $\omega_{j}$ occurring in (1.3), i.e. $q \geqslant m$. Another disadvantage is that a rather detailed knowledge of the dominant solution components is required. And even if this information is available, the frequencies $\omega_{j}$ may vary over one integration step (e.g. in non-linear problems) which will decrease the accuracy of these methods. Therefore, we are automatically led to the last approach of Table 1.

This third approach seems to be applicable to a fairly large class of problems. However, we have first to solve the minimax problem in which the $2 q$ parameters in $\phi$ are to be determined in such a way that $\max |\phi(i v)|$ is minimal in the interval $\underline{v} \leqslant v \leqslant \bar{v}$ (with $\underline{v}:=\omega h, \bar{v}:=\bar{\omega} h$ ). We will approximately solve this problem for small values of $\bar{v}-\underline{v}=(\bar{\omega}-\underline{\omega}) h$. Let the family of linear $k$-step methods containing the $2 q$ free coefficients be such that $\phi(z)=O\left(z^{r}\right), r \geqslant 1$. Then, for sufficiently small values of $\bar{v}-\underline{v}$, with $\underline{v} \neq 0$, we approximate $|\phi(i v)|$ by

$$
|\phi(i v)| \approx v^{r} P_{2 q}(v) \approx\left(\frac{\bar{v}+\underline{v}}{2}\right)^{r} P_{2 q}(v), \quad v \in[\underline{v}, \bar{v}],
$$

where $P_{2 q}$ is a polynomial of degree $2 q$ in $v$ assuming non-negative values in the interval $[\nu, \bar{v}]$. Adopting the validity of the approximation (2.6) we now define $P_{2 q}(v)$ such that it has a minimal maximum norm in the interval $[\nu, \bar{v}]$. If the approximation (2.6) would be exact then such a minimax polynomial would be optimal. Thus, the value of this third approach depends on the validity of the approximation (2.6).

The minimax polynomials $P_{2 q}$ can be determined by exploiting the so-called "equal ripple property" satisfied by such polynomials, that is $P_{2 q}$ assumes alternatingly equal minimum and equal maximum values in the interval $[y, \bar{v}]$. Now consider the shifted Chebyshev polynomial

$$
\alpha\left[1+T_{2 q}\left(\frac{2 v-\bar{v}-\underline{v}}{\bar{v}-\underline{v}}\right)\right], \quad T_{2 q}(x):=\cos [2 q \arccos x],
$$

where $\alpha$ is an approximate constant. It is easily verified that this polynomial satisfies the equal ripple property in $[v, \bar{v}]$ so that the minimax polynomial $P_{2 q}(v)$ is of this form. As a consequence, $P_{2 q}(v)$ has the $q$ (double) zeros

$$
v^{(l)}:=\frac{1}{2}(\bar{v}+\underline{v})+\frac{1}{2}(\bar{v}-\underline{v}) \cos \frac{2 l-1}{2 q} \pi, \quad l=1,2, \ldots, q .
$$

The free coefficients in the function $\phi$ are therefore determined by the (linear) system

$$
\phi\left(i v^{(l)}\right)=0, \quad l=1,2, \ldots, q .
$$

Obviously, for $h=0$ the methods defined by (2.7) reduce to a "conventional" linear multistep method, that is all zeros of the function $\phi(z)$ are located at $z=0$ if $h=0$ (notice that the coefficients in $\phi(z)$ depend on $\underline{v}$ and $\bar{v}$, and therefore on $h$ ). Since $\phi(z)$ 
has $r$ zeros at $z=0$ (by assumption) and $q$ double zeros at $z=i v^{(l)}, l=1,2, \ldots, q$, we have a zero of order $r+2 q$ at $z=0$ if $h=0$. Thus, the method resulting from the third approach is of (algebraic) order $p=r+2 q-1$.

In this paper we concentrate on methods satisfying the minimax conditions (2.7). These methods will be called minimax methods. In particular we will consider the minimax methods generated by

$$
\begin{aligned}
& \left\{k=5, \rho(\zeta)=\zeta^{5}-\zeta^{4}, r=1, q=3, \sigma(\zeta) \quad \text { determined by }(2.7 \mathrm{a}) \text { and }(2.7 \mathrm{~b})\right\}, \\
& \left\{k=5, \rho(\zeta)=\zeta^{5}-\zeta^{3}, r=1, q=3, \sigma(\zeta) \quad \text { determined by }(2.7 \mathrm{a}) \text { and }(2.7 \mathrm{~b})\right\},(2.8) \\
& \left\{k=6,147 \sigma(\zeta)=60 \zeta^{6}, r=1, q=3, \rho(\zeta) \quad \text { determined by (2.7a) and }(2.7 \mathrm{~b})\right\} .
\end{aligned}
$$

These methods will be denoted by $A M_{6}(\underline{v}, \bar{v}), M S_{6}(\underline{v}, \bar{v})$ and $B D_{6}(\underline{v}, \bar{v})$, respectively. For $h \rightarrow 0$ they successively converge to the well-known sixth order AdamsMoulton $\left(A M_{6}\right)$, Milne-Simpson $\left(M S_{6}\right)$ and backward differentiation $\left(B D_{6}\right)$ method. These conventional methods are characterized by

$$
\begin{aligned}
& A M_{6}: \quad \rho(\zeta)=\zeta^{5}-\zeta^{4} ; \quad 1440 \sigma(\zeta)=475 \zeta^{5}+1427 \zeta^{4}-798 \zeta^{3}+482 \zeta^{2}-173 \zeta+27, \\
& M S_{6}: \quad \rho(\zeta)=\zeta^{5}-\zeta^{3} ; \quad 90 \sigma(\zeta)=28 \zeta^{5}+129 \zeta^{4}+14 \zeta^{3}+14 \zeta^{2}-6 \zeta+1, \\
& B D_{6}: 147 \rho(\zeta)=147 \zeta^{6}-360 \zeta^{5}+450 \zeta^{4}-400 \zeta^{3}+225 \zeta^{2}-72 \zeta+10 ; \quad 147 \sigma(\zeta)=60 \zeta^{6} \text {, }
\end{aligned}
$$

respectively.

Crucial for the accuracy behaviour of the minimax methods and of the conventional methods as well, is the maximum norm of the corresponding function $\phi(z)$, where $z \in[\underline{i}, i \bar{v}]$, i.e.

$$
M(\underline{v}, \bar{v} ; q):=\max _{\underline{v} \leqslant v \leqslant \bar{v}}|\phi(i v)| .
$$

For several $(\underline{v}, \bar{v})$-intervals we calculated the $M(\underline{v}, \bar{v} ; 3)$-values for the minimax methods (2.8) and compared them with $M(0, \bar{v} ; 0)$, which determines the local truncation error of the corresponding conventional method. In Table 2 we give these $M(0, \bar{v} ; 0)$-values for the methods (2.9), while Table 3 contains the gain factors

TABLE 2

$M(0, \bar{v} ; 0)$-values for the conventional methods (2.9)

\begin{tabular}{cccc}
\hline $\bar{v}$ & \multicolumn{1}{c}{$A M_{6}$} & \multicolumn{1}{c}{$M S_{6}$} & \multicolumn{1}{c}{$B D_{6}$} \\
\hline $0 \cdot 05$ & $0 \cdot 11.10^{-10}$ & $0 \cdot 76 \cdot 10^{-11}$ & $0 \cdot 46 \cdot 10^{-10}$ \\
$0 \cdot 10$ & $0 \cdot 14 \cdot 10^{-8}$ & $0 \cdot 98 \cdot 10^{-9}$ & $0 \cdot 58 \cdot 10^{-8}$ \\
$0 \cdot 15$ & $0 \cdot 24 \cdot 10^{-7}$ & $0 \cdot 17 \cdot 10^{-7}$ & $0 \cdot 99.10^{-7}$ \\
\hline
\end{tabular}

TABLE 3

$M(0, \bar{v} ; 0) / M(\underline{v}, \bar{v} ; 3)$ factor for the minimax methods (2.8)

\begin{tabular}{cccc}
\hline $\bar{v}$ & $\underline{\nu}=0$ & $\underline{v}=0 \cdot 05$ & $\underline{v}=0 \cdot 10$ \\
\hline $0 \cdot 05$ & 10 & $\infty$ & \\
$0 \cdot 10$ & 10 & 48 & $\infty$ \\
$0 \cdot 15$ & 10 & 24 & 140 \\
\hline
\end{tabular}


$M(0, \bar{v} ; 0) / M(\underline{v}, \bar{v} ; 3)$ for the minimax methods $(2.8)$. These factors turned out to be constant for the several types of methods.

\section{Stability}

Concerning the stability of the linear multistep methods we followed the usual approach as can be found in e.g. Lambert (1973). In Fig. 1 are plotted (parts of) the stability regions of the $A M_{6}, M S_{6}$ and $B D_{6}$ method (we mention that the regions are symmetric about the real axis).

The stability regions of the corresponding minimax methods for realistic $(\underline{v}, \bar{v})$-values (say $\underline{v} \leqslant \bar{v} \leqslant 0 \cdot 1$ ) are very similar to the ones given in Fig. 1. It turned out that there are no points on the imaginary axis for which the sixth order MilneSimpson method is absolutely stable.

In connection with stability we mention a paper by Skelboe \& Christensen (1981) in which the stability regions of the BD methods are enlarged by appending two exponential terms to the polynomial basis of the classical formulae.

\section{Numerical Comparisons}

In this section the minimax methods generated by (2.8) are compared with the corresponding conventional linear multistep methods and with the methods based on the approach of Gautschi, that is, if $\omega_{0}$ is an estimate of the frequency of the exact local solution (cf. (2.4)) and $v_{0}=\omega_{0} h$ then these methods are also based on (2.8) in which the condition (2.7a) is replaced by $v^{(l)}=l v_{0}, l=1, \ldots, q$. These methods will be indicated by $A M_{6}\left(v_{0}\right), M S_{6}\left(v_{0}\right)$ and $B D_{6}\left(v_{0}\right)$, respectively. In both cases the linear system (2.7b) (to obtain the coefficients for the linear multistep

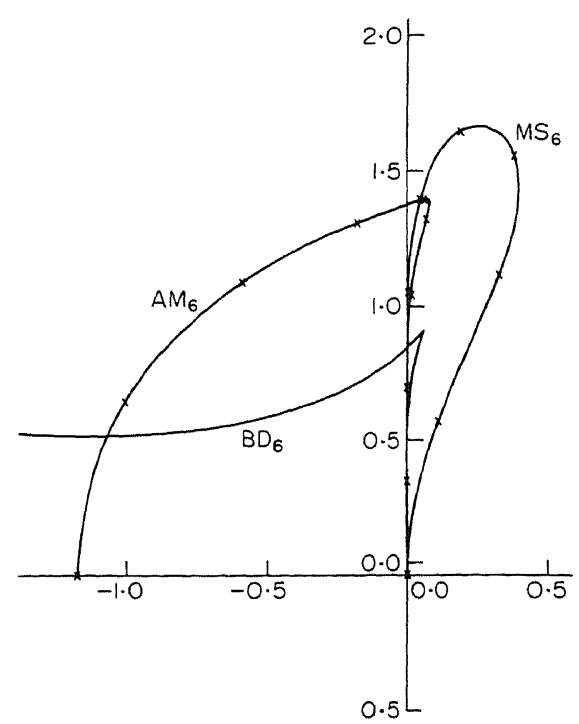

Fig. 1. Stability regions of the $A M_{6}, M S_{6}$ and $B D_{6}$ method. 
methods) was solved numerically. However, if the $v^{(l)}$-values are nearly equal this system is very ill-conditioned and we ran into numerical problems. In that case we changed to the system

$$
\left.\frac{d^{j}}{d z^{j}} \phi(z)\right|_{z=\frac{1}{2} i(\psi+i)}=0, \quad j=0,1, \ldots, q-1 .
$$

In all experiments the starting values were taken from the exact solution or from a sufficiently accurate reference solution. The implicit relations were solved using Newton iteration. All problems were converted to their first-order equivalents and for measuring the obtained accuracy we used the number of correct significant decimals in the end point $t_{\text {end }}$ of the integration, i.e.

$$
s d:=\log _{10}\left(L_{2}-\text { norm of the error at } t_{\text {end }}\right) \text {. }
$$

The calculations were performed an a CDC CYBER 175-750 which has a 48-bit mantissa yielding a machine precision of about 14 decimal digits.

Finally, we deliberately tried to select problems which are illustrative for the various kinds of difficulties we wanted to test for. The particular difficulty is mentioned in each subsection.

\subsection{Periodic Solutions}

Consider the sixth order model differential equation

$$
\left\{\prod_{j=1}^{3}\left(\frac{d^{2}}{d t^{2}}+\omega_{j}\right)\right\} y(t)=0, \quad 0 \leqslant t \leqslant 12 \pi=t_{\text {end }} ; \quad \omega_{j} \geqslant 0
$$

with the exact solution

$$
y(t)=\sum_{j=1}^{3}\left(C_{j}^{+} e^{i \omega_{j} t}+C_{j}^{-} e^{-i \omega_{j} t}\right),
$$

where the constants $C_{j}^{ \pm}$are determined by the initial conditions.

Choosing

$$
C_{j}^{+}=(1-i) / 2, \quad C_{j}^{-}=(1+i) / 2, \quad \omega_{1}=0 \cdot 7, \quad \omega_{2}=\frac{2 \cdot 8}{3}, \quad \omega_{3}=1 \cdot 4
$$

we have the solution

$$
y(t)=\sum_{j=1}^{3}\left(\sin \left(\omega_{j} t\right)+\cos \left(\omega_{j} t\right)\right)
$$

which is periodic with frequency

$$
\omega_{0}=\frac{0.7}{3}=0.2333 \ldots
$$

Applying the several methods we obtained the results as listed in Table 4. For this linear model problem, the theory of Section 2 is confirmed rather well.

We repeated the experiment but now the frequency $\omega_{2}$ was changed to 0.9 . The solution is no longer periodic in the interval of integration, but we can regard it as "almost" periodic with frequency $\omega_{0} \approx 0 \cdot 23$. The results obtained differ only slightly 
TABLE 4

Results obtained for problem (4.2)-(4.4)

\begin{tabular}{|c|c|c|c|c|c|c|c|c|c|}
\hline$h$ & $A M_{6}$ & $M S_{6}$ & $B D_{6}$ & $A M_{6}\left(\frac{0 \cdot 7}{3} h\right)$ & $M S_{6}\left(\frac{0 \cdot 7}{3} h\right)$ & $B D_{6}\left(\frac{0 \cdot 7}{3} h\right)$ & $A M_{6}(0 \cdot 7 h, 1 \cdot 4 h)$ & $M S_{6}(0 \cdot 7 h, 1 \cdot 4 h)$ & $B D_{6}(0 \cdot 7 h, 1 \cdot 4 h)$ \\
\hline$\pi / 10$ & 1.44 & 1.97 & $0 \cdot 41$ & 1.62 & $2 \cdot 13$ & 0.59 & $3 \cdot 12$ & $3 \cdot 56$ & 2.09 \\
\hline$\pi / 25$ & $3 \cdot 86$ & $4 \cdot 32$ & $2 \cdot 85$ & 4.05 & $4 \cdot 51$ & 3.04 & $5 \cdot 54$ & $6 \cdot 00$ & $4 \cdot 35$ \\
\hline$\pi / 50$ & $5 \cdot 66$ & $6 \cdot 12$ & $4 \cdot 66$ & $5 \cdot 85$ & $6 \cdot 31$ & $4 \cdot 85$ & $7 \cdot 34$ & $7 \cdot 80$ & $6 \cdot 34$ \\
\hline
\end{tabular}

TABLE 5

Results for problem (4.7), (4.8)

\begin{tabular}{|c|c|c|c|c|c|c|c|c|c|}
\hline$h$ & $A M_{6}$ & $M S_{6}$ & $B D_{6}$ & $A M_{6}(10 h)$ & $M S_{6}(10 h)$ & $B D_{6}(10 h)$ & $A M_{6}(9 \cdot 9 h, 10 \cdot 1 h)$ & $M S_{6}(9 \cdot 9 h, 10 \cdot 1 h)$ & $B D_{6}(9 \cdot 9 h, 10 \cdot 1 h)$ \\
\hline $1 / 25$ & $2 \cdot 27$ & $2 \cdot 02$ & 1.05 & $4 \cdot 50$ & $4 \cdot 51$ & $3 \cdot 32$ & $7 \cdot 20$ & $5 \cdot 66$ & $6 \cdot 42$ \\
\hline $1 / 50$ & 4.57 & $5 \cdot 14$ & $3 \cdot 24$ & $6 \cdot 89$ & $6 \cdot 80$ & $5 \cdot 56$ & $8 \cdot 60$ & $8 \cdot 73$ & $7 \cdot 74$ \\
\hline $1 / 100$ & $6 \cdot 38$ & $6 \cdot 73$ & $5 \cdot 49$ & $8 \cdot 46$ & 8.88 & $7 \cdot 66$ & $10 \cdot 30$ & $10 \cdot 77$ & $9 \cdot 30$ \\
\hline
\end{tabular}


from the results of Table 4 (there was no difference in $s d$-values found, greater than 0.03).

\section{Conclusions:}

The change from a periodic solution to an "almost" periodic solution has no significant influence on the accuracy of the results.

The methods have some benefit from the Gautschi approach; however, a substantial gain in accuracy is obtained by minimizing the local truncation error on the $\omega$-interval $[0 \cdot 7,1 \cdot 4]$.

Making a mutual comparison between the methods, the Milne-Simpson method seems to be the most accurate one for this problem (cf. Table 2).

\subsection{Uncertainty in the Periodicity}

Next, we test the problem (cf. Gautschi, 1961; Neta \& Ford, 1984)

$$
\ddot{y}(t)+\left(100+\frac{1}{4 t^{2}}\right) y(t)=0, \quad 1 \leqslant t \leqslant 10,
$$

with the initial values according to the "almost" periodic particular solution

$$
y(t)=\sqrt{t} J_{0}(10 t),
$$

where $J_{0}$ is a Bessel function of the first kind. Clearly, the frequency of this "almost" periodic solution is close to 10 and therefore we applied the Gautschi-methods with $\omega_{0}=10$. However, this problem is an example for which the spectrum of the Jacobian matrix gives detailed information about the local behaviour of the solution. A straightforward calculation reveals that the eigenvalues $\omega$ are approximately given by $\omega_{ \pm}(t) \approx \pm 10 i\left[1+1 /\left(800 t^{2}\right)\right]$. Hence, we applied our minimax methods with $\underline{\omega}=9.9$ and $\bar{\omega}=10 \cdot 1$.

Table 5 shows the results of the various methods. Compared with the conventional methods there is a gain in accuracy of about two decimal digits in favour of the Gautschi approach.

The minimax methods, however, have a further increase in accuracy of about two decimal digits.

Finally, we anticipate that the accuracy can be still more increased by exploiting the special structure of the second order differential equation, i.e. the absence of the first order derivative. For, the ideas of minimizing the function $\phi$ on a suitable interval can analogously be applied to linear multistep methods which are designed for this type of equation, e.g. Störmer type methods.

\subsection{Non-imaginary Noise}

In this subsection we want to test, apart from over- or underestimating the frequency of the solution, the influence of non-imaginary noise. By this, we mean 
that the local solution contains not only oscillatory components but also some "noise", caused by non-imaginary eigenvalues of the Jacobian matrix, i.e.

$$
y(t)=\sum_{j} c_{j} e^{i \omega_{j} t}+\delta z(t)
$$

For that purpose we selected the orbit equation (cf. Hull, Enright, Fellen \& Sedgwick, 1972, Problem Class D)

with solution

$$
\begin{gathered}
\ddot{u}(t)+u(t) / r^{3}=0, \quad u(0)=1-\varepsilon, \quad \dot{u}(0)=0, \\
\ddot{v}(t)+v(t) / r^{3}=0, \quad v(0)=0, \quad \dot{v}(0)=((1+\varepsilon) /(1-\varepsilon))^{\frac{1}{2}}, \\
r^{2}=u^{2}(t)+v^{2}(t), \quad 0 \leqslant t \leqslant 12 \pi
\end{gathered}
$$

$$
\begin{gathered}
u(t)=\cos \tau-\varepsilon, \quad \dot{u}(t)=-\sin \tau /(1-\varepsilon \cos \tau), \\
v(t)=\left(1-\varepsilon^{2}\right)^{\frac{1}{2}} \sin \tau, \quad \dot{v}(t)=\left(1-\varepsilon^{2}\right)^{\frac{1}{2}} \cos \tau /(1-\varepsilon \cos \tau),
\end{gathered}
$$

where $\tau-\varepsilon \sin \tau=t$ ( $\varepsilon$ is the eccentricity of the orbit).

The initial conditions correspond to a local solution of the form (4.9) in which $\delta$ is small.

First, we concentrate on an adequate treatment of the oscillatory part of the solution. For $\varepsilon=0$, the complex eigenvalues of the Jacobian matrix are $\pm i$. However, for a non-zero eccentricity $\varepsilon$ they are time-dependent and hard to determine in advance. For $\varepsilon=0.01$ we integrated this problem twice:

(i) the estimate of the frequency of the solution is 1 , that is the Gautschiapproach was applied with $\omega_{0}=1$ and the minimax methods employed the $\omega$-interval $[0 \cdot 9,1 \cdot 1]$. The results are given in Table 6 .

(ii) secondly, $\omega_{0}=0.9$ in the Gautschi approach and the $\omega$-interval $[0.8,1]$ for the minimax methods was used. Table 7 shows the results of this experiment.

From these tables we see a dramatic drop in accuracy for the Gautschi methods when the frequency is wrongly estimated by only a small percentage.

Again, the minimax methods show that they perform equally well in both cases and do not need an accurate foreknowledge of the frequency of the solution.

Let us return to the real subject of this subsection. The first part of the right-hand side of (4.9) is properly treated by the minimax methods (cf. (1.3)), the second part is not. If $h$ decreases, the influence of this term on the accuracy increases, hence the minimax methods gradually lose their superiority, as can be seen in Table 7. This effect is pronounced in the experiment with an eccentricity $\varepsilon=0 \cdot 1$, the results of which can be found in Table 8 .

\subsection{Stiff Components}

So far, the $B D_{6}$ methods turned out to be inferior to the $A M_{6}$ and $M S_{6}$ methods as far as accuracy was concerned. In this subsection we will illustrate the use of $B D_{6}$ methods when the exact local solution is of the form (cf. (1.3))

$$
y(t) \approx c_{0}+\sum_{j=1}^{m_{1}} c_{j} e^{i \omega_{j} t}+\sum_{j=1}^{m_{2}} d_{j} e^{-\hat{\omega}_{j} t},
$$

where the $\hat{\omega}_{j}$ are positive and large (the so-called stiff components). 
TABLE 6

Results for problem (4.10), (4.11) ( $(\varepsilon=0.01)$, with $\omega_{0}=1 \cdot 0, \underline{\omega}=0.9$ and $\bar{\omega}=1 \cdot 1$

\begin{tabular}{cccccccccc}
\hline$h$ & $A M_{6}$ & $M S_{6}$ & $B D_{6}$ & $A M_{6}(h)$ & $M S_{6}(h)$ & $B D_{6}(h)$ & $A M_{6}(0 \cdot 9 h, 1 \cdot 1 h)$ & $M S_{6}(0 \cdot 9 h, 1 \cdot 1 h)$ & $B D_{6}(0 \cdot 9 h, 1 \cdot 1 h)$ \\
\hline$\pi / 10$ & 1.46 & 0.56 & 0.27 & 6.32 & 3.56 & 4.59 & $2 \cdot 76$ & $1 \cdot 21$ & 1.86 \\
$\pi / 25$ & 4.34 & 3.09 & 3.08 & 7.68 & 5.69 & 6.73 & $5 \cdot 01$ & 3.69 & 4.04 \\
$\pi / 50$ & 6.81 & 5.08 & 5.33 & $9 \cdot 42$ & 7.66 & 8.85 & $6 \cdot 79$ & 5.68 & $5 \cdot 80$ \\
\hline
\end{tabular}

TABLE 7

Results for problem (4.10), (4.11) ( $(\varepsilon=0.01)$, with $\omega_{0}=0 \cdot 9, \underline{\omega}=0.8$ and $\bar{\omega}=1 \cdot 0$

\begin{tabular}{cccccccccc}
\hline$h$ & $A M_{6}$ & $M S_{6}$ & $B D_{6}$ & $A M_{6}(0 \cdot 9 h)$ & $M S_{6}(0 \cdot 9 h)$ & $B D_{6}(0 \cdot 9 h)$ & $A M_{6}(0 \cdot 8 h, h)$ & $M S_{6}(0 \cdot 8 h, h)$ & $B D_{6}(0 \cdot 8 h, h)$ \\
\hline$\pi / 10$ & 1.46 & 0.56 & 0.27 & 0.94 & 0.74 & -0.24 & $2 \cdot 70$ & $1 \cdot 13$ & 1.80 \\
$\pi / 25$ & $4 \cdot 34$ & 3.09 & 3.08 & 3.73 & 3.06 & 2.55 & 4.94 & 3.62 & 3.97 \\
$\pi / 50$ & 6.81 & 5.08 & 5.33 & 5.84 & 5.01 & 4.65 & $6 \cdot 71$ & 5.61 & $5 \cdot 73$ \\
\hline
\end{tabular}

TABLE 8

Results for problem (4.10), (4.11) $(\varepsilon=0 \cdot 1)$, with $\omega_{0}=0 \cdot 9, \omega=0.8$ and $\bar{\omega}=1 \cdot 0$

\begin{tabular}{|c|c|c|c|c|c|c|c|c|c|}
\hline$h$ & $A M_{6}$ & $M S_{6}$ & $B D_{6}$ & $A M_{6}(0 \cdot 9 h)$ & $M S_{6}(0 \cdot 9 h)$ & $B D_{6}(0 \cdot 9 h)$ & $A M_{6}(0 \cdot 8 h, h)$ & $M S_{6}(0 \cdot 8 h, h)$ & $B D_{6}(0 \cdot 8 h, h)$ \\
\hline$\pi / 10$ & $1 \cdot 10$ & -0.64 & 0.09 & 0.90 & $0 \cdot 31$ & -0.25 & 1.71 & -0.47 & 0.78 \\
\hline$\pi / 25$ & 3.63 & $1 \cdot 61$ & $3 \cdot 28$ & $3 \cdot 81$ & $2 \cdot 11$ & 2.58 & 3.62 & 1.73 & $2 \cdot 83$ \\
\hline$\pi / 50$ & $5 \cdot 14$ & $3 \cdot 61$ & $4 \cdot 25$ & $6 \cdot 34$ & 4.09 & $4 \cdot 87$ & $5 \cdot 25$ & $3 \cdot 73$ & $4 \cdot 31$ \\
\hline
\end{tabular}


TABLE 9

Results of the $B D_{6}$ methods for problem (4.13), (4.14) with $\omega_{0}=1$, $\underline{\omega}=0.9$ and $\bar{\omega}=1 \cdot 1$

\begin{tabular}{cccc}
\hline$h$ & $B D_{6}$ & $B D_{6}(h)$ & $B D_{6}(0 \cdot 9 h, 1 \cdot 1 h)$ \\
\hline $1 / 10$ & 5.40 & 6.08 & $7 \cdot 34$ \\
$1 / 25$ & $7 \cdot 76$ & 8.44 & $9 \cdot 51$ \\
\hline
\end{tabular}

Apart from the initial phase, these components hardly influence the oscillatory behaviour of the solution but they demand a highly stable method. For example, we see from Fig. 1 that the step size in the $A M_{6}$ method should satisfy $h \leqslant 1 \cdot 18 / \hat{\omega}$, $\hat{\omega}=\max _{j} \hat{\omega}_{j}$, and that the $M S_{6}$ method is absolutely unstable for every $h$. However, in case of the $B D_{6}$ method, the value of $\hat{\omega}$ does not impose a restriction on the step size (see also Lambert, 1973).

Let us consider the problem

$$
\begin{aligned}
& \dddot{y}(t)+(2 \varepsilon y(t)-\lambda) \ddot{y}(t)+\left(1+\varepsilon^{2} y^{2}(t)-2 \varepsilon \lambda y(t)\right) \dot{y}(t)-\lambda\left(1+\varepsilon^{2} y^{2}(t)\right) y(t)= \cos t, \\
& 0 \leqslant t \leqslant 20
\end{aligned}
$$

with initial conditions

$$
y(0)=1, \quad \dot{y}(0)=1, \quad \ddot{y}(0)=-1 .
$$

For small values of $\varepsilon$, the eigenvalues of the Jacobian matrix are approximately given by $\pm i$ and by $\lambda$. In our experiment we choose $\varepsilon=10^{-2}, \lambda=-100$ and determined a reference solution with an explicit Runge-Kutta method using a very small step size. The results of the $B D_{6}$ methods are given in Table 9. For the step sizes of this table the $A M_{6}$ and $M S_{6}$ methods behaved unstably. Again, the minimax method is superior to the Gautschi-approach and to the conventional method.

The authors are indebted to the referee for carefully reading the manuscript and for several suggestions which improved the paper.

\section{REFERENCES}

BETTIS, D. G. 1970 Numerical integration of products of Fourier and ordinary polynomials. Num. Math. 14, 421-434.

GAUTSCHI, W. 1961 Numerical integration of ordinary differential equations based on trigonometric polynomials. Num. Math. 3, 381-397.

Hull, T. E., Enright, W. H., Fellen, B. M. \& SedGwick, A. E. 1972 Comparing numerical methods for ordinary differential equations. SIAM J. num. Analysis 9, 603-637.

Lambert, J. D. 1973 Computational Methods in Ordinary Differential Equations. London: Wiley.

LiNigER, W. \& WilloughBy, R. A. 1970 Efficient integration methods for stiff systems of ordinary differential equations. SIAM J. num. Analysis 7, 47-66.

LYCHE, T. 1972 Chebyshevian multistep methods for ordinary differential equations. Num. Math. 19, 65-75.

NetA, B. \& Ford, C. H. 1984 Families of methods for ordinary differential equations based on trigonometric polynomials. JCAM 10, 33-38.

SKelboe, S. \& CHRISTENSEN, B. 1981 Backward differentiation formulas with extended regions of absolute stability. BIT 21, 221-231.

Stiefel, E. \& BeTtis, D. G. 1969 Stabilization of Cowell's method. Num. Math. 13, 154-175. 